\title{
Stone extraction with loop ureteral catheter versus ureteroscopy in small distal ureteral stones - retrospective comparison of 547 consecutive patients
}

\author{
Christian Rehme ${ }^{1}$, Carolin Burchert ${ }^{2}$, Mustafa Tosun ${ }^{1}$, Tibor Szarvas ${ }^{1}$, Nadine Nagy ${ }^{3}$, \\ Herbert Ruebben $^{1,4}$, Boris Hadaschik ${ }^{1}$, Christian Niedworok ${ }^{1,2}$ \\ ${ }^{1}$ Department of Urology, University Hospital Essen, University of Duisburg-Essen, Essen, Germany; ${ }^{2}$ Department of Urology, Hermann-Josef \\ Hospital Erkelenz, University of Duisburg-Essen, Essen, Germany; ${ }^{3}$ Division of Infectious Diseases and Geographic Medicine, Department of \\ Medicine, Stanford University School of Medicine, Stanford, CA, USA; ${ }^{4}$ Department of Urology, Helios Hospital Duisburg, Duisburg, Germany \\ Contributions: (I) Conception and design: All authors; (II) Administrative support: C Niedworok, B Hadaschik, T Szarvas; (III) Provision of study \\ materials or patients: All authors; (IV) Collection and assembly of data: All authors; (V) Data analysis and interpretation: C Niedworok, B Hadaschik, \\ T Szarvas; (VI) Manuscript writing: All authors; (VII) Final approval of manuscript: All authors. \\ Correspondence to: Privatdozent Dr. med, Christian Niedworok. Department of Urology, University Hospital Essen, University of Duisburg-Essen, \\ Hufelandstrasse 55, 45147 Essen, Germany. Email: christian.niedworok@uni-due.de.
}

Background: To evaluate whether stone extraction with a loop ureteral catheter (LUC) in distal ureteral stones is associated with a higher frequency of ureteral strictures compared to treatment with primary ureteroscopic stone removal (p-URS) or ureteroscopic laser lithotripsy (l-URS).

Methods: Five hundred and forty-seven consecutive patients were primarily endourologically treated for distal ureteral stones in our department between 2005 and 2019 and included in the study protocol. Data was retrospectively obtained from the patients' charts and medical reports as well as from office-based urologists. Data analysis was performed using Fisher's exact test, Mann-Whitney test or Student's $t$-test as appropriate. A level of $\mathrm{P}<0.05$ was assigned statistical significance.

Results: Four hundred and twelve patients were treated by URS (p-URS n=304, 1-URS n=108) and another 135 by LUC stone extraction. Median follow-up was 41 [2-159] months. There was no difference between the groups concerning age, gender, proportion of patients with ureteral stenting, operating time, hospitalization or readmission rates. The number of ureteric strictures was small in all procedures $[\mathrm{n}=3(1.0 \%)$ in $\mathrm{p}$-URS, $\mathrm{n}=2(1.9 \%)$ in $1-\mathrm{URS}$ and $\mathrm{n}=2(1.5 \%)$ in LUC] and there was no difference between the groups concerning this serious complication (p-URS vs. LUC: $\mathrm{P}=0.6465$; 1-URS vs. LUC: $\mathrm{P}=0.9999$ ).

Conclusions: In small distal stones, LUC stone extraction still is an alternative to URS procedures in stone management with comparable results concerning postinterventional ureteral strictures. In experienced hands, it still has its value in accurately selected patients.

Keywords: Ureteral calculus; urolithiasis; ureter; ureteroscopy (URS); loop catheter

Submitted May 03, 2020. Accepted for publication Sep 28, 2020.

doi: 10.21037/tau-20-909

View this article at: http://dx.doi.org/10.21037/tau-20-909

\section{Introduction}

Urolithiasis is a very widespread disease and one of the most common diseases in urology. The lifetime risk for urolithiasis in developed countries is quantified to be $1-13 \%$ (1). Nine point seven percent males have already been diagnosed with urolithiasis in an age between 50 and 69 years. With $42 \%$, the recurrence rate of urolithiasis is very high (2). Urolithiasis is associated with numerous metabolic disorders and suspected to be a risk factor for life threatening diseases like ischemic strokes, myocardial 


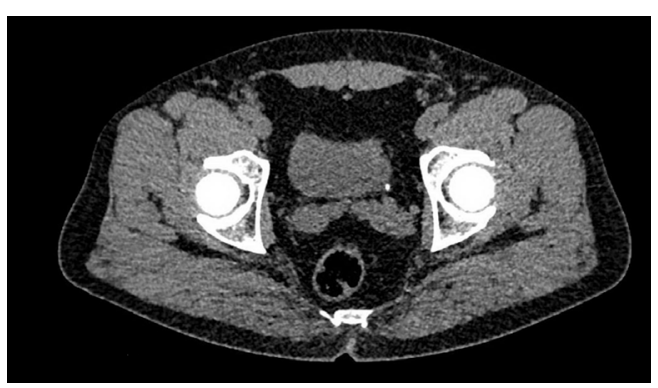

Figure 1 CT-scan of a 28 -year-old male patient with a small distal ureteral stone. The patient was referred to our department with acute pain of the lower abdomen. His ultrasound examination showed a slight dilatation of the ipsilateral renal pelvis. The present CT scan showed a prevesical ureteral stone $(2 \times 3 \mathrm{~mm}$, 853 Hounsfield Units). After excluding a UTI, the patient was treated by a loop catheter stone extraction and the stone was completely removed. He was discharged the next day without complications and follow-up investigation showed no further problems. UTI, urinary tract infection.

infarction and further cardiovascular events $(3,4)$. The current European Association of Urology (EAU) guideline recommendations for small ureteral stones include observation and periodic evaluation as a treatment option as long as the stone size does not exceed $10 \mathrm{~mm}$ and a spontaneous stone passage can be expected $(5,6)$. If an endourological approach is intended, the stone removal should be performed under endoscopic visualization (7). Only controlled means of manipulation in the upper urinary tract are recommended to avoid ureteric strictures after stone removal. To date, ureteroscopy (URS) is the most favored endourological stone treatment in ureterolithiasis. Loop ureteral catheter (LUC) guided stone extraction was established in 1937 by Zeiss with high complication rates. In older series, severe complications such as ureter perforation or postinterventional strictures resulting in the need of surgical lithotomy were described in up to $47 \%$ of cases (8). At least no difference concerning the stone free rates was shown in a comparison between URS and LUC guided stone extraction (9). In the period prior to the utilization of semirigid or flexible endoscopes, stone extraction with the looped probe often was used as the only alternative for lithotomy. As a result of lacking alternatives, loop guided stone removal was quite commonly carried out (8-14).

In our department, URS is the preferred method for removal of ureteral stones, but LUC extraction still has its value for small distal stones. In the present study, we compared the clinical outcome and complications in patients either treated with URS or LUC extraction of small distal ureteral stones.

We present the following article in accordance with the STROBE guideline checklist (available at http://dx.doi. org/10.21037/tau-20-909).

\section{Methods}

We identified 4.978 consecutive patients that were treated for urolithiasis at our department between 2009 and 2019. Of these, 1.997 were diagnosed with a ureteral stone. A total amount of 561 patients could be identified with a singular stone of $\leq 10 \mathrm{~mm}$ diameter in the distal part of the ureter, in detail below the crossover of the common iliac arteries. The present study included those 547 consecutive patients that were endourologically treated. In the remaining 14 cases, extracorporeal shockwave lithotripsy (ESL) was performed. Due to the small amount of ESL procedures, we excluded the patients treated with this method.

The primary endpoint of this study was the rate of postoperative ureteric strictures. All patients were followed from baseline (date of surgery) until July 2019. Clinical and pathological data was obtained from patients' medical reports in the outpatient's department and from the patient's general practitioners and office-based urologists. The patients were contacted to evaluate the duration of ureteral stenting and whether further interventions were performed as a result of complications from manipulation in the upper urinary tract. Written informed consent was obtained from the patients for publication of this Original Article. The study was conducted in accordance with the Declaration of Helsinki (as revised in 2013). Patients were treated according to the Treatment Guidelines of the European Association of Urology. The Committee for Research of the Faculty of Medicine, University Hospital Essen, University Duisburg-Essen approved the study protocol (ID 20-9191-BO).

\section{Techniques}

All patients were diagnosed with a pre-interventional CTscan or abdominal X-ray (Figure 1). Maximum stone size for primary stone extraction without disintegration was $10 \mathrm{~mm}$. All patients were offered a conservative medical treatment before interventional therapy, except those patients suffering from severe pain, concomitant urinary 

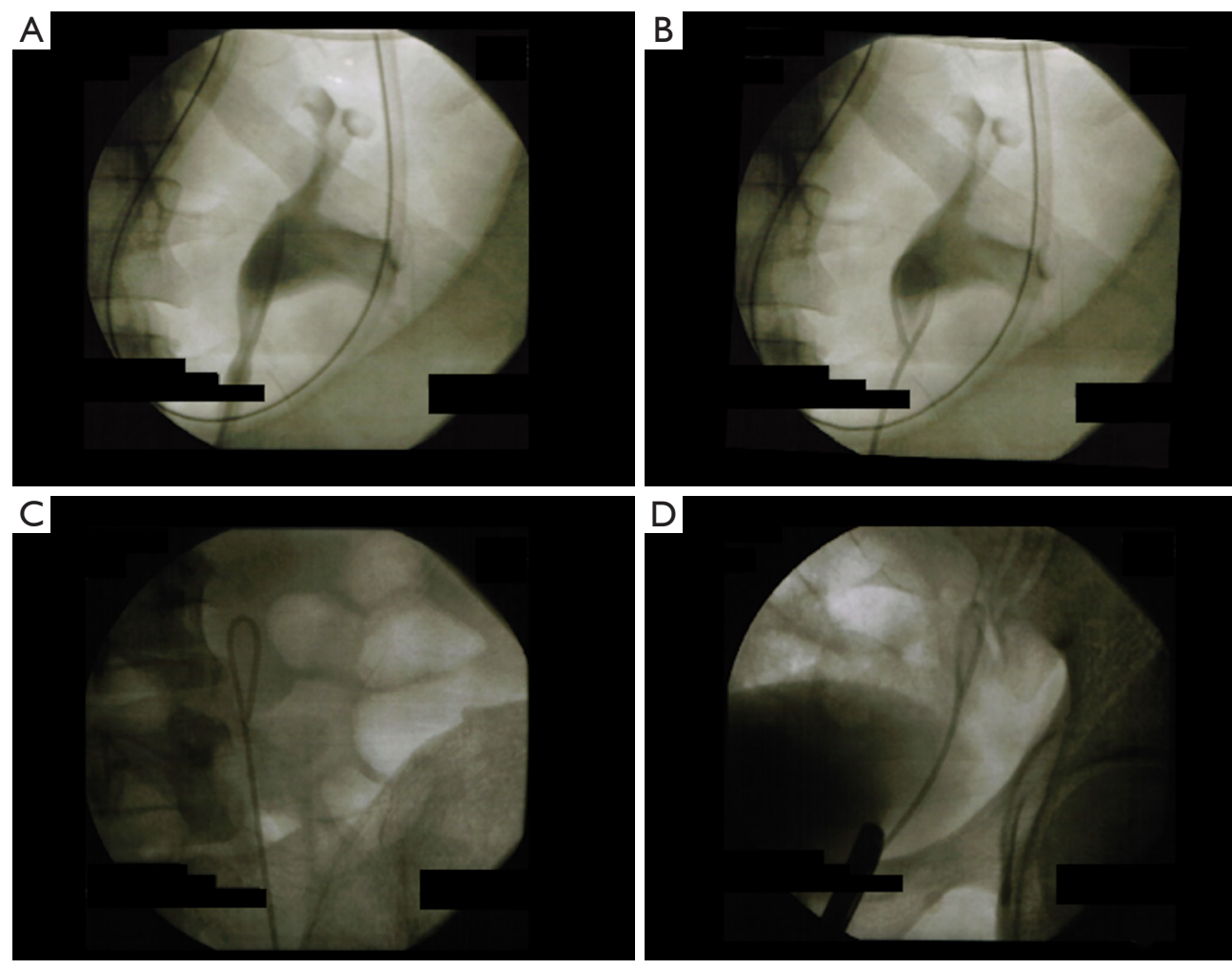

Figure 2 Intraoperative radiographs during loop guided extraction of a distal ureter stone. A LUC is inserted into the left ureteral orifice under radiographic control (A). After closure of the extraction loop at the tip of the catheter in the renal pelvis (B), the catheter is gently retracted under radiographic guidance (C,D). LUC, loop ureteral catheter.

tract infection (UTI) or impairment of renal function who needed urgent treatment (6). The endourological treatment was performed by five different urologic surgeons with at least 7 years of surgical training. All procedures were carried out under general anesthesia. According to the decision of the treating urologic surgeon, either a LUC extraction or a URS was performed. All patients were offered single-shot antibiotic prophylaxis or antibiotic therapy according to the results of the preoperative urine culture.

Catheter stone extraction was done using a 5 French loop catheter (Coloplast ${ }^{\circledR}$, Humlebaek, Denmark). First, the ureter and renal pelvis were visualized by retrograde urography and the stone localization was assessed. In larger stones ( $\geq 5 \mathrm{~mm}$ ), a guidewire was inserted into the ureter. Then the loop catheter was inserted and guided into the ureter under radiographic control. When the catheter was placed in the renal pelvis, the loop was closed and gently retracted to the ureteral orifice under radiographic control (Figures 2,3). When stone removal was successful, a final retrograde urography was performed. In cases when loop catheter extraction was not successful, the catheter was returned into the renal pelvis and then removed with the loop straightened up to avoid ureteral injuries.

URS was performed using flexible (URF-P5 5.3 French tip, Olympus ${ }^{\circledR}$, Tokyo, Japan) or rigid (OES-Pro 6.4 French tip, Olympus ${ }^{\circledR}$, Tokyo, Japan) instruments. The instrument was inserted into the ureteral orifice under visual control after putting a guidewire into the ureter. The stone was removed using a nitinol basket. If a laser lithotripsy was performed, a 30W Holmium:YAG laser (Dornier ${ }^{\circledR}$ MedTech GmbH, Wessling, Germany) was used.

At the end of the treatment, as described above, a retrograde urography was performed and if needed, a ureter catheter inserted. The indication for ureteral stenting based on the surgeons decision. A stent was inserted when there was reduced drainage of contrast medium from the upper urinary tract visualized during a final retrograde urography at the end of the procedure. Stent removal was performed in an office-based setting, performing cystoscopy in local anesthesia. 

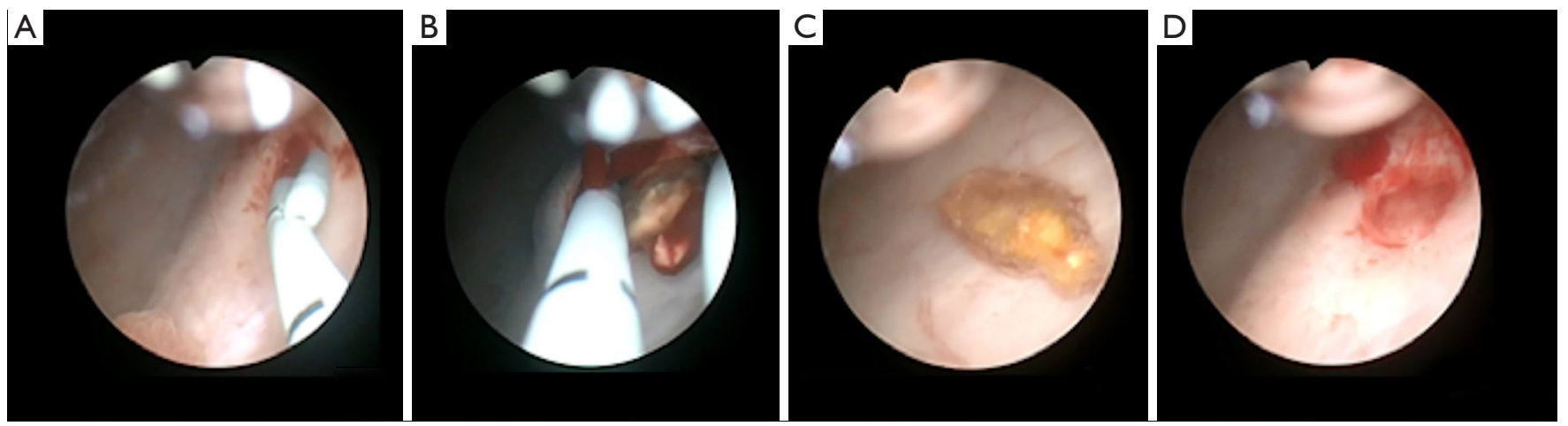

Figure 3 Once the stone is entangled by the loop (A), it can be removed from the ureteral orifice under careful manual traction (B). The stone $(2 \times 4 \mathrm{~mm}$ calcium oxalate) is located in the bladder at the end of the extraction $(\mathrm{C})$ and can be picked with a transurethral forceps. Aspect of the ureter orifice at the end of the procedure: the orifice is wide open and the mucosal ridge in good condition (D).

\section{Follow-up and evaluation of strictures}

All 547 patients that were identified with a singular urolithiasis of the distal ureter were contacted. Between March and July 2019, the patients were contacted by mail or by telephone. If there was no response from the patients, the attending general practitioners or office-based urologists were contacted. Age, gender, stone size, ureteral stenting, duration of ureteral stenting and hospitalization, operating time, dilatation of the upper urinary tract, appearance of ureteral stricture and the readmission rates within 3 months after stone removal were assessed. In total, 451 replies could be evaluated, representing a response rate of $82.4 \%$. During follow-up, patients were evaluated for upper urinary tract dilatation with routinely performed ultrasound examinations during the first 6 months after stone removal. For 3 years, annually and thereafter symptom-based examinations were performed. Depending on the findings, further imaging examinations were coordinated if necessary.

\section{Statistical analysis}

Data are presented as medians \pm SEM. Statistical significance was assigned at the level of $\mathrm{P}<0.05$. Data lacking normal distribution were analyzed by the non-parametric two-tailed Wilcoxon rank-sum test (Mann-Whitney) for paired group comparisons. Proportional distribution of the postoperative findings were analyzed using the Fisher's exact test. Difference between two groups was assessed using Student's $t$-test as appropriate. Analyses were done using the IBM $^{\circledR}$ SPSS $^{\circledR}$ software (version 24.0, Chicago, IL, USA) and GraphPad Prism ${ }^{\circledR}$ (version 6, La Jolla, CA, USA).

\section{Results}

\section{Study population}

From the 547 patients with distal ureteral stones, 412 were treated by URS and another 135 by LUC. In 304 of the URS cases, the stone was primarily removed with a nitinol basket [primary ureteroscopic stone removal (p-URS)] and in 108 cases a laser lithotripsy was performed before removal of the fragments. Median follow-up was 41 [2-159] months. The largest average stone size was $4.8[1-10] \mathrm{mm}$ in the ureteroscopic laser lithotripsy (l-URS) group, compared to 4.4 [1-10] $\mathrm{mm}$ in the p-URS and 4.0 [1-10] $\mathrm{mm}$ in the LUC group (p-URS vs. 1-URS: $\mathrm{P}=0.1305$; $\mathrm{p}$-URS vs. LUC: $\mathrm{P}=0.1071$; l-URS vs. LUC: $\mathrm{P}=0.0086)$. There was no difference between the URS groups and the LUC group concerning median patients' age \{p-URS: 49 [19-86] y, l-URS: 49 [17-84] y; LUC: 45 [6-83] y; $\mathrm{P}=0.2140\}$. There also was no difference in the amounts of ureteral stenting (p-URS: 57.9\%; 1-URS: 61.1\%; LUC: $59.3 \%$ ), operating time \{p-URS: 44 [25-104] min; 1-URS: 46 [31-96] min; LUC: $45[32-70] \mathrm{min}$ \} or duration of hospitalization \{p-URS: 5.2 [3-12] d; 1-URS: 5.4 [3-12] d; LUC: 5.0 [3-9] d]. Longest median duration of ureteral stenting was seen in the in the LUC group 17 [1-40] days compared to 16 [0-41] days in the p-URS and also 16 [1-38] days in the 1-URS group ( $\mathrm{p}$-URS vs. 1-URS: $\mathrm{P}=0.6920$; $\mathrm{p}$-URS $v s$. LUC: $\mathrm{P}=0.0132$; 1 -URS vs. LUC: $\mathrm{P}=0.1310)$. In all three groups male patients were predominant $(75.7 \%$ p-URS; $67.6 \%$ 1-URS; 77.0\% LUC) (Tables 1,2). Stone free rates in first attempt were $91.4 \%$ in p-URS, $91.7 \%$ in l-URS and $92.6 \%$ in LUC. 
Table 1 Patients' age, stone size, duration of ureteral stenting, follow-up, duration of hospitalization and operating time in the three groups

\begin{tabular}{|c|c|c|c|c|c|}
\hline Parameters & All $(n=547)$ & $\begin{array}{l}\text { Ureterrenoscopy and primary } \\
\text { stone removal }(n=304)\end{array}$ & $\begin{array}{l}\text { Ureterrenoscopy and } \\
\text { laser lithotripsy }(n=108)\end{array}$ & $\begin{array}{l}\text { Loop ureter catheter } \\
\qquad(n=135)\end{array}$ & $P$ \\
\hline Age, years & & & & & 0.2140 \\
\hline Stone size, $\mathrm{mm}$ & & & & & 0.0320 \\
\hline Average [range] & $4.4[1-10]$ & $4.4[1-10]$ & $4.8[1-10]$ & $4.0[1-10]$ & \\
\hline Median [range] & $16[0-41]$ & $16[0-41]$ & $16[1-38]$ & $17[1-40]$ & \\
\hline Follow-up, months & & & & & - \\
\hline Median [range] & 41 [2-159] & 40 [2-159] & 39 [4-139] & 54 [2-149] & \\
\hline Average [range] & $5.2[3-12]$ & $5.2[3-12]$ & 5.4 [3-12] & $5.0[3-9]$ & \\
\hline
\end{tabular}

Table 2 Comparison of gender, amount of patients with ureteral stenting, dilatation of the upper urinary tract during follow-up, rate of ureteric strictures and proportion of readmissions within 3 months after stone removal in the different groups

\begin{tabular}{|c|c|c|c|c|c|c|c|}
\hline Parameters & All $(n=547)$ & $\begin{array}{l}\text { A: ureterrenoscopy and } \\
\text { primary stone removal }(n=304)\end{array}$ & $\begin{array}{l}\text { B: ureterrenoscopy and } \\
\text { laser lithotripsy }(n=108)\end{array}$ & $\begin{array}{l}\text { C: loop ureter } \\
\text { catheter }(n=135)\end{array}$ & \multicolumn{3}{|c|}{$\mathrm{P}$} \\
\hline Gender, n (\%) & & & & & 0.1271 & 0.8090 & 0.1116 \\
\hline Male & 407 (74.4) & $230(75.7)$ & 73 (67.6) & $104(77.0)$ & & & \\
\hline Female & $140(75.6)$ & 74 (24.3) & $35(32.4)$ & $31(23.0)$ & & & \\
\hline Yes & 322 (58.9) & $176(57.9)$ & $66(61.1)$ & $80(59.3)$ & & & \\
\hline No & $225(41.1)$ & $128(42.1)$ & $42(38.9)$ & $55(40.7)$ & & & \\
\hline $\begin{array}{l}\text { Dilatation durin } \\
\text { follow-up, n (\%) }\end{array}$ & & & & & 0.5968 & 0.1914 & 0.4106 \\
\hline Yes & $7(1.0)$ & $3(1.0)$ & $2(1.9)$ & $2(1.5)$ & & & \\
\hline No & $540(99.0)$ & 301 (99.0) & $106(98.1)$ & $133(98.5)$ & & & \\
\hline $\begin{array}{l}\text { Readmission <3 mo, } \\
\mathrm{n}(\%)\end{array}$ & & & & & 0.8393 & 0.5537 & 0.6140 \\
\hline No & 407 (74.4) & $280(92.1)$ & $99(91.7)$ & $127(94.1)$ & & & \\
\hline Yes & $140(75.6)$ & $24(7.9)$ & $9(8.3)$ & $8(5.9)$ & & & \\
\hline
\end{tabular}




\section{Complications}

There was no difference in the proportion of readmissions within 3 months after stone removal (p-URS: 7.9\%; l-URS: 8.3\%; LUC: $5.9 \%$ ). There was no difference between the groups in the amount of postoperative upper urinary tract dilatations (p-URS: 4.3\%; l-URS: 5.6\%; LUC: $2.2 \%$ ). There also was no difference in the amount of ureteral strictures in the different groups (p-URS: $1.0 \%$; 1-URS: $1.9 \%$; LUC: $1.5 \%$ ) (Table 2). In each group there was one ureteric perforation which was treated by insertion of a ureteral stent for 2 weeks.

In two patients with $5 \mathrm{~mm}$ stones we initially tried LUC stone extraction, but the shape of the stones did not allow the attempts. We converted to URS and rotated the stones in a parallel axle to the ureter. A basket stone extraction could then be performed without complications.

Fortunately, ureteric stricture was a rare complication and could be identified in three patients in the p-URS group, two patients in the 1-URS and also two patients in the LUC group ( $1.3 \%$ for the whole cohort). One of the patients with a ureteric stricture in the p-URS group was a 45 -year-old man who was diagnosed with urolithiasis for the first time. During the intervention, a $6 \mathrm{~mm}$ stone was removed from the intramural part of the prevesical ureter on the right-hand side. At the end of the procedure a retrograde urography was performed and showed a good urine flow throughout the ureteral orifice with a completely emptied upper urinary tract. The next morning, we had to insert a ureteral stent due to severe flank pain and a dilatation of the upper urinary tract. The stent was removed after 2 weeks and initially there was no further complaint. After another 4 weeks, the patient was readmitted due to recurrent flank pain and the diagnostic workup showed a long segment ureter stricture $(5 \mathrm{~cm})$ of the distal ureter. An endoscopic approach did not seem promising, so the distal ureter was resected and a ureterocystoneostomy (psoas hitch technique) was performed.

One of the patients with a postinterventional ureteric stricture in the LUC group was a 32-year-old man with a first-time urolithiasis as well. His $6 \mathrm{~mm}$ stone in the prevesical part of the ureter was removed via LUC and the retrograde urography showed no evidence of upper urinary tract dilatation. After 6 weeks, the patient was referred to our department again due to dilatation of the ipsilateral ureter. Endourological evaluation showed a $5 \mathrm{~mm}$ ring-shaped stricture $3 \mathrm{~cm}$ before the ureteral orifice. We treated the stricture using a holmium laser (Dornier $^{\circledR}$
MedTech GmbH, Medilas H20, Wessling, Germany) with a $200 \mu \mathrm{m}$ laser fibre and insertion of a 7 French ureter stent for 3 weeks. After removing the stent, the patient was symptomfree and no further dilatation of the upper urinary tract was seen. In the remaining five patients, ureterocystoneostomy was performed in three further cases. One patient was treated by insertion of a permanent metal ureteral stent and one patient is treated by regularly changes of the inserted ureteral stent ("double-J"). Table 3 displays a summary of the patients with postinterventional ureteric strictures.

\section{Discussion}

In modern medicine, there is an overall impression that good medical treatment is naturally associated with technical effort and that a high-end technique must be superior to cost-effective solutions. However, LUC guided stone extraction was shown previously to be an equally effective procedure compared to URS with a stone free rate of $87-93 \%$. Highest stone free rates are achieved in small distal ureteral stones (9). Today, stone removal with a LUC has almost been completely replaced by endoscopic techniques. In the present study we show that ureteric stricture as a feared complication of stone treatment is not common in patients treated with a LUC for distal ureteral stones in carefully selected patients.

URS is the standard of care treatment for ureteral stones. Its effectiveness and low rates of perioperative morbidity are striking arguments for a broad usage in stone management $(9,10)$. Understandably, no endoscopic urologist longs to regain times when stone extraction was done with a LUC that was left under continuous traction but without control in the ureter for hours or even several days $(11,12)$. Severe complications with major ureteral injuries in $7-47 \%$ are described in older studies, when the patients' selection was not adequate and distal ureter stones as well as proximal ureter stones or stones in the renal calices were treated with a LUC under continuous traction $(8,13,14)$. However, in a conscientious selection of patients, a catheter guided stone extraction can be a useful addition to the technical portfolio in stone management. Before decision for a stone removal with a LUC, there should be an adequate diagnostic imaging workup to define the amount, size and density of calculi in the urinary tract. Patients with more than a solitary stone are not suitable for LUC stone extraction and should therefore be treated with another appropriate endoscopic technique. This also applies to situations, when the stone is not localized in the distal part of the ureter. 


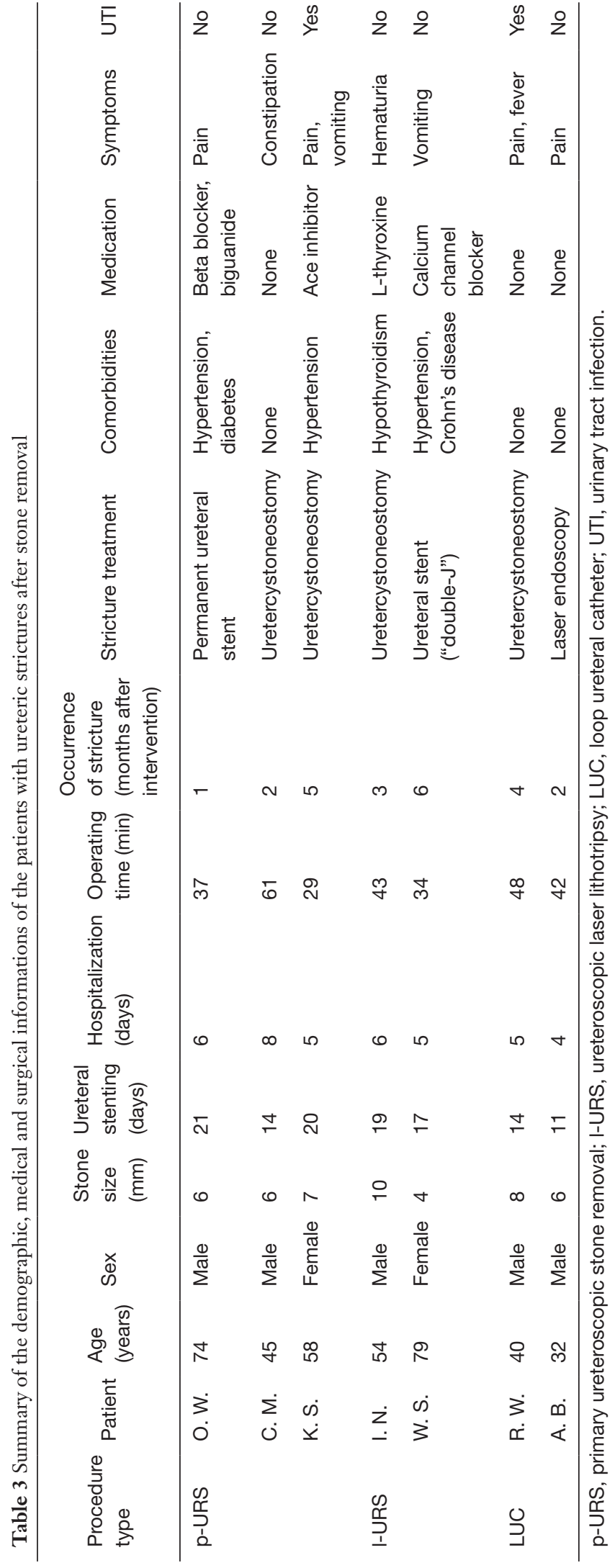

Decision for either loop guided stone removal or URS was taken by the surgeon during the ongoing procedure. Decision making based upon experience with the different techniques respecting appearance of the ureteral orifice, width and course of the ureter and the flexibility of the lower ureter.

Before stone extraction, UTI must be excluded to avoid severe complications like urosepsis. In an older series of 342 patients, two patients died during LUC stone extraction due to complications following the treatment (14). We recommend a single shot antibiotic application before endoscopic stone treatment to prevent perioperative fever, regardless from the preoperative urine culture results. In cases with a verified UTI, we recommend antibiotic therapy as appropriate. We implemented this approach in all our patients which is in line with the current European guidelines recommendations $(6,7)$. The approach for LUC guided stone extraction should only be repeated once in the same procedure. If the stone was not removed within the first two attempts, we changed the technique and converted to stone extraction with URS.

In our patient cohort, there were seven cases of ureteric strictures ( $1.3 \%$ for the whole cohort), equally distributed over the different treatment groups. This was in line with the results of a large multi-institutional series including 9,681 patients with urolithiasis. In 4,479 cases, a distal ureteral stone was diagnosed and the treatment resulted in ureter obstruction or stricture and the need for further treatment in 83 cases (1.9\%) (10). In our study in all cases with a ureteric stricture, further interventional treatment was necessary to handle this serious long-lasting complication. In all our cases, the patients were fine in the end, four of them after performing a ureterocystoneostomy, one after an endoscopic laser treatment. In two cases the physical condition of the patients were critical for surgery. As a consequence, a long-term metal stent insertion was performed in one case and a long-term ureter stent ("double-J") insertion was performed in another case.

An important aspect in this context is the postoperative aftercare. In our department, patients are asked to visit their office-based urologist at least 2 weeks after they were discharged from the hospital and again after a period of 6 months. All patients were advised to get an annually ultrasound examination for the next 3 years after stone removal. This is in line with the results of observational studies that recommend follow-up periods from $3-18$ months $(10,15-18)$. If there are any complaints or if there are suspicious findings in the aftercare examinations, 
the patients are referred back to our department for further evaluation. This ensures the diagnosis of ureteric strictures in a timely manner and the above-mentioned patients were treated without significant loss of their renal function, as we checked in renal function scintigraphy.

In our opinion, even though stone removal with a LUC is a rarely used technique, it has its value in accurately selected patients. We suggest small distal ureter stones in patients without UTI as indication of this technique. When used in experienced hands, a loop guided procedure can be part of technical diversity in modern stone treatment.

Limitations of the present study are derived from its retrospective character. Selection of patients for a particular procedure was depending on the surgeon's experience and preference. This could result in a selection bias as the choice of the urologic surgeon for a special procedure is a subjective parameter. The choice of the technique was also influenced by stone morphometry, the impression of the upper urinary tract during retrograde urography and the impression of the ureteral orifice during endoscopy. All these aspects have the potential for selection bias. As asymptomatic upper urinary tract dilatation can occur after the suggested long-term follow-up, these patients might be underrepresented in our cohort.

\section{Conclusions}

Our results show that a catheter extraction of small distal ureteral stones may have its value in certain circumstances. In well selected patients, severe complications are not common in the treatment of small distal ureteral stones with a LUC.

\section{Acknowledgments}

Funding: None.

\section{Footnote}

Reporting Checklist: The authors have completed the STROBE reporting checklist. Available at http://dx.doi. org/10.21037/tau-20-909

Data Sharing Statement: Available at http://dx.doi. org/10.21037/tau-20-909

Peer Review File: Available at http://dx.doi.org/10.21037/ tau-20-909
Conflicts of Interest: All authors have completed the ICMJE uniform disclosure form (available at http://dx.doi. org/10.21037/tau-20-909). Dr. BH reports grants, personal fees and non-financial support from Jansen, personal fees and non-financial support from AstraZeneca, personal fees and non-financial support from Bayer, personal fees and non-financial support from Astellas, grants from German Cancer Aid, other from Uromed, personal fees from Lightpoint Medical, personal fees from ABX, outside the submitted work. The other authors have no conflicts of interest to declare.

Ethical Statement: The authors are accountable for all aspects of the work in ensuring that questions related to the accuracy or integrity of any part of the work are appropriately investigated and resolved. Written informed consent was obtained from the patients for publication of this Original Article. The study was conducted in accordance with the Declaration of Helsinki (as revised in 2013). Patients were treated according to the Treatment Guidelines of the European Association of Urology. The Committee for Research of the Faculty of Medicine, University Hospital Essen, University DuisburgEssen approved the study protocol (ID 20-9191-BO). Confidentiality of the patients' data was guaranteed and all data was anonymized. Future management of the patients is not affected by the present study. There was no study funding by external third parties. The study was initiated, conducted, financed and analyzed by the team of authors.

Open Access Statement: This is an Open Access article distributed in accordance with the Creative Commons Attribution-NonCommercial-NoDerivs 4.0 International License (CC BY-NC-ND 4.0), which permits the noncommercial replication and distribution of the article with the strict proviso that no changes or edits are made and the original work is properly cited (including links to both the formal publication through the relevant DOI and the license). See: https://creativecommons.org/licenses/by-nc-nd/4.0/.

\section{References}

1. Sorokin I, Mamoulakis C, Miyazawa K, et al. Epidemiology of stone disease across the world. World J Urol 2017;35:1301-20.

2. Raheem OA, Khandwala YS, Sur RL, et al. Burden of urolithiasis: trends in prevalence, treatments, and costs. Eur Urol Focus 2017;3:18-26. 
3. Lin SY, Lin CL, Chang YJ, et al. Association between kidney stones and risk of stroke: a nationwide populationbased cohort study. Medicine (Baltimore) 2016;95:e2847.

4. Alexander RT, Hemmelgarn BR, Wiebe N, et al. Kidney stones and cardiovascular events: a cohort study. Clin J Am Soc Nephrol 2014;9:506-12.

5. Preminger GM, Tiselius HG, Assimos DG, et al. 2007 guideline for the management of ureteral calculi. J Urol 2007;178:2418-34.

6. Türk C, Petřík A, Sarica K, et al. EAU guidelines on diagnosis and conservative management of urolithiasis. Eur Urol 2016;69:468-74.

7. Türk C, Petř́k A, Sarica K, et al. EAU Guidelines on Interventional Treatment for Urolithiasis. Eur Urol 2016;69:475-82.

8. Ipiéns-Aznar A. Delivery of low ureteric stones with the Zeiss indwelling probe. Br J Urol 1982;54:223-5.

9. Börgermann C, vom Dorp F, Sperling H, et al. The Zeissloop stone extraction: minimal invasive and effective or obsolete? World J Urol 2010;28:187-92.

10. Perez Castro E, Osther PJ, et al. Differences in ureteroscopic stone treatment and outcomes for distal, mid-, proximal, or multiple ureteral locations: the Clinical Research Office of the Endourological Society ureteroscopy global study. Eur Urol 2014;66:102-9.

Cite this article as: Rehme C, Burchert C, Tosun M, Szarvas T, Nagy N, Ruebben H, Hadaschik B, Niedworok C. Stone extraction with loop ureteral catheter versus ureteroscopy in small distal ureteral stones-retrospective comparison of 547 consecutive patients. Transl Androl Urol 2021;10(1):87-95. doi: 10.21037/tau-20-909
11. Lynn JM, Chamberlain NO, Cannon RD, et al. Loop catheter extraction of ureteral stones: a re-evaluation. Urology 1983;22:243-5.

12. Karcher G. Removal of ureteral calculi transurethrally with special reference to the so-called "indwelling Zeiss loop" (author's transl). Urologe A 1981;20:269-73.

13. Bowers L. Loop catheter delivery of ureteral calculi. J Urol 1973;110:178-80.

14. Nagel R, Marquardt H, Grull S. Treatment of ureteral calculi with the Zeiss loop. Int Urol Nephrol 1972;4:215-20.

15. Baş O, Tuygun C, Dede O, et al. Factors affecting complication rates of retrograde flexible ureterorenoscopy: analysis of 1571 procedures-a single-center experience. World J Urol 2017;35:819-26.

16. Darwish AE, Gadelmoula MM, Abdelkawi IF, et al. Ureteral stricture after ureteroscopy for stones: a prospective study for the incidence and risk factors. Urol Ann 2019;11:276-81.

17. Fam XI, Singam P, Ho CC, et al. Ureteral stricture formation after ureteroscope treatment of impacted calculi: a prospective study. Korean J Urol 2015;56:63-7.

18. de la Rosette J, Denstedt J, Geavlete P, et al. The clinical research office of the endourological society ureteroscopy global study: indications, complications, and outcomes in 11,885 patients. J Endourol 2014;28:131-9. 